\title{
Short-Term Wind Power Forecasting Using Empirical Mode Decomposition and RBFNN
}

\author{
Zeng-Wei Zheng ${ }^{\mathrm{a}}$, Yuan-Yi Chen ${ }^{\mathrm{a}, \mathrm{b}}$, Xiao-Wei Zhou ${ }^{\mathrm{a}}$, Mei-Mei Huo ${ }^{\mathrm{a} *}$, \\ Bo Zhao ${ }^{c}$, Min-Yi Guo ${ }^{\mathrm{d}}$ \\ ${ }^{a}$ Department of Computer, Zhejiang University City College, Hangzhou 310015, China \\ ${ }^{b}$ College of Computer Science and Technology, Zhejiang University, Hangzhou 310027, China \\ ${ }^{c}$ Institute of Zhejiang Electric Power Test \& Research, Hangzhou 310014, China \\ ${ }^{d}$ College of Computer Science and Technology, Shanghai Jiao Tong University, Shanghai 200240, China
}

\begin{abstract}
In order to effectively predict wind farm power with non-linear and non-stationary characteristics, a prediction model based on empirical mode decomposition (EMD) and radial basis function neural networks(RBFNN) was designed. The forecast model uses EMD to decompose the wind power into several intrinsic mode functions (IMF) and one residue. The RBFNN was used to construct a prediction model for each IMF component and the residue, the input variables of the prediction model are triple: wind speed, wind direction, and history wind power. All the prediction results of components were aggregated to obtain the ultimate prediction result. The simulation results show that compared with the traditional prediction method based on artificial neural networks, this method has high prediction precision and strong adaptability.
\end{abstract}

Keywords: Wind power prediction, empirical mode decomposition, radial basis function neural networks, Kalman filter, least squares method

\section{Introduction}

Today, wind power has become an important component of smart grid and it will meet $12 \%$ of global electricity demand by 2020[1]. Due to the characteristics of wind (unstable, intermittent and uncontrollable, etc.), the integration of wind power plants with traditional power grid suffer serious challenges and so restrict the scale of wind power development. One of the most important issues solving this problem is to accurately predict for wind power[2-4].

For this reason, a lot of wind power forecast methods have been proposed in recent years. These prediction methods can be divided into two categories: A. Prediction methods based on historical data. The basic idea is to construct a mapping between historical wind farm data (such as power, wind speed, wind direction, etc) and future power output. These forecast methods include: Kalman filter method [5], Least squares method [6], random statistical autocorrelation moving average ARMA (p, q) method [7], kernel density estimation [8], adaptive fuzzy logic algorithm [9] and others. B. Prediction methods based on numerical weather prediction. The main idea is using numerical weather provided by the meteorological department to predict weather conditions (including wind speed, wind direction, air temperature, air pressure and other parameters) of wind farms, and then put these parameters as the input of forecast model to get the predictive value of power. These methods include: a combination prediction model of neuro-fuzzy and artificial neural network [10], artificial neural network [11], adaptive fuzzy inference system [12], complex-valued neural network [13], recurrent high order neural networks [14] and so on. Due to the strong complexity, non-linear and non-stationary characteristics of wind power, it is

\footnotetext{
* Manuscript received June 12, 2012; revised August 28, 2012.

Corresponding author. Tel.: +86-571-88018813; E-mail address: huomeimei@zucc.edu.cn.
} 
very difficult to improve prediction accuracy for these traditional forecasting methods.

A reasonable approach to improve prediction accuracy would be to decompose the wind power and parse out the components which represent its changeable characteristics, and then forecast according to the regularity of each component is a reasonable approach. EMD is a method of breaking down a signal without leaving the time domain. The process is useful for analyzing natural signals, especially for nonlinear and non-stationary signals, compared to other analysis methods like Fourier Transforms and Wavelet Decomposition. Using EMD to decompose non-stationary and non-linear signal into several intrinsic mode functions (IMF) with different oscillation periods, despite some components still being non-linear and non-stationary to a degree, the mutual influence among them has been isolated. Therefore, using this isolation can reduce the predicted impacts of non-stationary and non-linear behavior and so improve prediction accuracy.

This paper attempts to combine EMD and radial basis function neural networks (RBFNN) to forecast short-term wind power. The process is as follows: first, to use EMD to decompose wind power into several IMFs with different characteristic scales and a residue and to analyze the regularity of these IMFs and the residue; and secondly, to build RBFNN forecast models for each IMF and residue and aggregative all the predictive values of IMFs and the residue to obtain the final predictive value.

\section{Empirical Mode Decomposition Principles}

Empirical mode decomposition (EMD) is a new method for analyzing non-linear and non-stationary signals, it was proposed by Norden E. The Huallg et al. in 1998 [15]. The decomposition process is based on the following assumptions: 1)the signal has at least a maximum value and a minimum value; 2)the interval between extreme values is in characteristic time degrees; 3)if a signal only has an inflection point not extreme point, the extreme point can be obtained by one or several differential calculation, and get the decomposition result by integral calculation.

Using the EMD method, a signal can be decomposed into several intrinsic mode functions (IMF) and a residue. And each intrinsic mode function is defined by: 1) Equal number of extreme and zero crossings (at most more or less one); 2)The envelopes (defined by all the local maxima and minima) are symmetrical with respect to zero. This implies that the mean value of each IMF is zero.

Given a signal $x(t)$, the decomposition process is as follows:

1) Calculate all the local maxima and minima of $x(t)$. Interpolate between maxima and connect them by a cubic spline curve. The same applies for the minima in order to obtain the upper and lower envelopes $e_{\max }(t)$ and $e_{\min }(t)$, respectively.

2) Compute the mean value of the envelopes: $m(t)=\left(e_{\max }(t)+e_{\min }(t)\right) / 2$. And extract the detail $h_{1}(t)=x(t)-m_{1}(t)$

3) Check whether $h_{1}$ is an IMF(i.e. it satisfies the two conditions), if it is then $c_{1}=h_{1}$, and an IMF is derived; if $h_{1}$ is not an IMF, replace $x(t)$ with $h_{1}$, and repeat step (1)-(2) until it satisfies the stop conditions of an IMF.

4) Regard $r=x(t)-h_{1}$ as a new $x(t)$, the steps are applied repeatedly to the residue in order to obtain all the IMFs $c_{1}(t), \ldots, c_{n}(t)$ of the signal until a predetermined threshold is achieved, or until the residue becomes a monotonic function.

The result of the EMD process produces $N$ IMFs $\left(c_{1}(t), \ldots, c_{n}(t)\right)$ and a residue signal $r(t)$ :

$$
x(t)=\sum_{i=1}^{n} c_{i}(t)+r(t)
$$

where the first $c_{1}(t)$ extracted are the lower order IMFs which captures the fast oscillation modes while the last IMFs $c_{i}(t)$ produced are the higher order IMFs which represent the slow oscillation modes. The residue $r(t)$ reveals the general trend of the time series $x(t)$. 


\section{Forecast Model for Wind Power Farms}

Artificial neural network has been widely used in the prediction of non-linear systems due to its strong adaptability and learning ability. And many factors(such as wind speed, wind direction, air temperature, air pressure, etc) make wind power non-linear and non-stationary. The forecast results are often unsatisfactory when using neural network directly to predict. In order to further improve prediction accuracy, this paper use EMD to decompose wind power signal into several intrinsic mode functions (IMF) and a residue, despite some IMFs still being non-stationary to a degree, the mutual influence among them has been isolated. Therefore, using this isolation can reduce the predicted impact of nonstationary and non-linear behavior and then improve the prediction accuracy. According to the feature of each IMF to build RBFNN forecast models and then aggregate all the predictive values of IMFs and residue to obtain the final predictive value. And the structure of the forecasting model is shown in Fig. l.

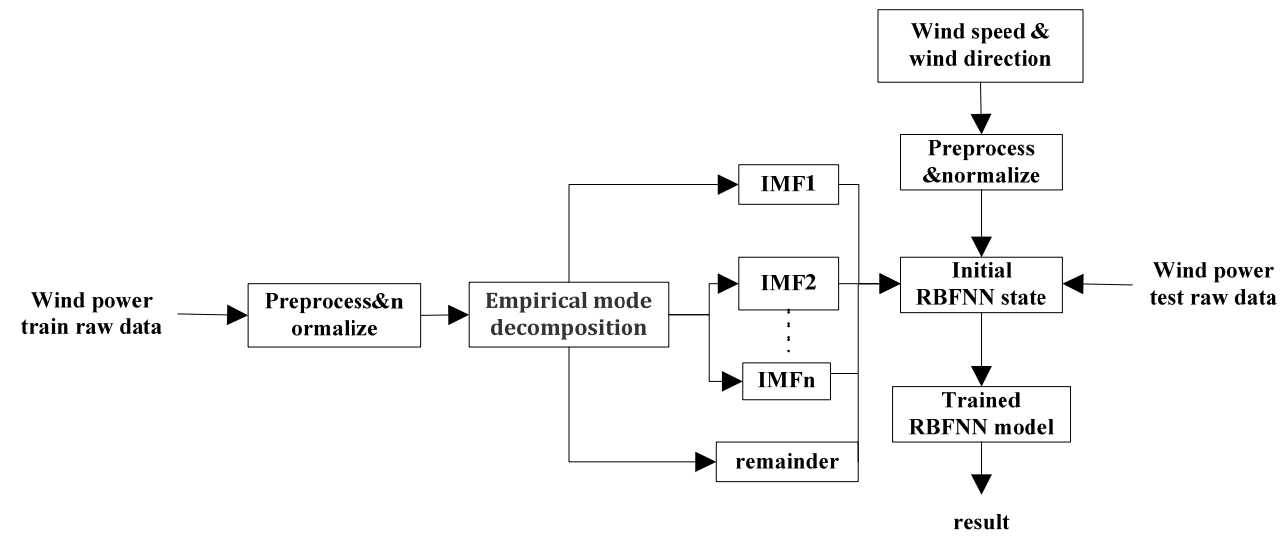

Fig. 1. Prediction process based on EMD and RBFNN.

\subsection{Preprocess and Normalization}

In our case, the wind farm has recorded detailed data sequentially even in the maintenance state or shut down by sudden gust, and thus, the raw data has not been helpful as such for the training of RBFNN. And the raw data need to pre-process to get rid of abundant or un-required data.

Kalman filter is used to eliminate noise[16]. However, the extracted data cannot be fed directly into the forecast model, because the range of the data has been very large while considering different operation parameters of the wind turbine. This problem has been solved by normalizing the data to uniform space mapping. In this paper, the data were normalized according to (2), whose values ranged from 0.0 to1.0.

$$
x^{l}(t)=\frac{x(t)-x_{\min }(t)}{x_{\max }(t)-x_{\min }(t)}
$$

where: $x^{l}(t)$ and $x(t)$ are time series data(wind power, wind speed and wind direction) non-normalized and normalized, respectively; and $x_{\max }(t)$ and $x_{\min }(t)$ are the maximum and the minimum absolute value of time series data, respectively.

Now there are a lot of samples and we need to choose a subset that contains the least number of key features contributing to accuracy while discarding the remaining unimportant features. The mutual information theory[17] which is based on entropy concept is used to select the subset and then use the subset as the input of the RBFNN model.

\subsection{Forecasting Method Based on RBFNN}

Radial basis function neural networks(RBFNN) was proposed by Moddy and Darken in 1988[18]. 
RBFNN is a kind of feed-forward neural network of three layers (input layer, hidden layer and output layer), it can approximate continuous function with any precision and particularly suit to solve classification and forecast problems. The basic idea is: to use radial basis function as a base unit to construct hidden layer, the input vector is directly mapped to hidden space without right connections. When radial basis function centre point is determined, then the mapping is identified.

Compared with other neural networks: (1)The output of RBFNN is the linear weighted sum of hidden unit output and its learning rate is faster; and (2)RBFNN use radial basis function (usually Gaussian function) as activation function, and neurons in the input space area are very small, so RBFNN need more radial basis neurons.

In the forecast model of this paper, the forecast model train algorithms as follows:

1) Network initialization. Randomly selected $h$ training samples as the clustering centre $c_{i}(k), i=1,2, \ldots, h$; and $k$ is the number of iteration. Calculate the distance between all the samples and cluster centres $\left\|x_{j}-c_{i}(k)\right\|, i=1,2, \ldots, h ; j=1,2, \ldots, n$.

2) All the input training samples grouped by nearest neighbour rule, when $i\left(x_{j}\right)=\min \left\|x_{j}-c_{i}(k)\right\| . i=1,2, \ldots, h ; j=1,2, \ldots, n . \quad x_{j}$ is classified as the $i$-th class, $x_{j} \in \theta_{i}(k), \theta_{i}(k)$ is cluster domain.

3) Adjust cluster centres.

$$
c_{i}(k+1)=\frac{1}{n_{i}} \sum_{x \in \theta_{i}(k)} x, i=1,2, \ldots, h
$$

where $n_{i}$ is the number of samples in $\theta_{i}(k)$.

4) if $c_{i}(k+1) \neq c_{i}(k)$, then go to step (2). Otherwise stop the cluster process.

The RBFNN choose Gaussian function as the radial basis function, so the variance can be calculated by formula(4):

$$
\sigma_{i}=\frac{c_{\max }}{\sqrt{2 h}}, i=1,2, \ldots, h
$$

where $C_{\max }$ is the max distance among all the selected cluster centres.

The neuron connection weights between hidden layer and output layer can be calculated by the least squares method[19]:

$$
w=\exp \left(\frac{h}{c_{\max }^{2}}\left\|x_{p}-c_{i}\right\|^{2}\right) p=1,2, \ldots, P ; i=1,2, \ldots, h
$$

where $c_{\max }$ is the max distance among selected cluster centers.

\subsection{Evaluation criteria}

In order to evaluate performance of forecast model in this paper, select the following four indicators as a basis of evaluation [15]:

1) Mean absolute error

$$
\text { MAE }=\frac{1}{N} \sum_{t=1}^{N}\left|y_{t}-y_{t}^{l}\right|
$$

2) Mean square error

$$
M S E=\frac{1}{N} \sqrt{\sum_{t=1}^{N}\left|y_{t}-y_{t}^{l}\right|^{2}}
$$

3) Mean absolute percent error 


$$
\text { MAPE }=\frac{1}{N} \sum_{t=1}^{N}\left|\frac{y_{t}-y_{t}^{l}}{y_{t}}\right| \times 100 \%
$$

4) Mean square percent error

$$
M S P E=\frac{1}{N} \sqrt{\frac{1}{N} \sum_{t=1}^{N}\left|\frac{y_{t}-y_{t}^{l}}{y_{t}}\right|} \times 100 \%
$$

where $y_{t}$ is the real wind farm power, $y_{t}^{l}$ is the predicted power, and $N$ is the test samples number for prediction model.

\section{Results and Analysis}

In this paper, the experimental data is from Zhejiang Provincial Electric Power Test Research Institute. The output power of a wind turbine is selected to verify the proposed forecast model. The power output is sampled per $60 \mathrm{~min}$ in this model, and chose 974 points to analyse. The actual wind turbine output power time series has some abnormal data, Kalman filter is used to eliminate noise. The de-noised wind turbine output power curve is shown in Fig. 2.

Fig. 3 shows the IMFs and residue decomposed by empirical mode decomposition method, with all of the IMFs ordered by frequency from high to low.

It can be observed from Fig. 3, that IMF1 fluctuates frequently and the variation is not obvious. It reflects the high frequency part of wind power and can be seen as a small random interference, and it is excluded in the forecast. IMF7 and IMF8 are the low frequency component and their cycle is big. The residue RES represents the wind power trend, and its mean value is close to the mean value of the overall wind power, reflecting the overall trend of wind power.

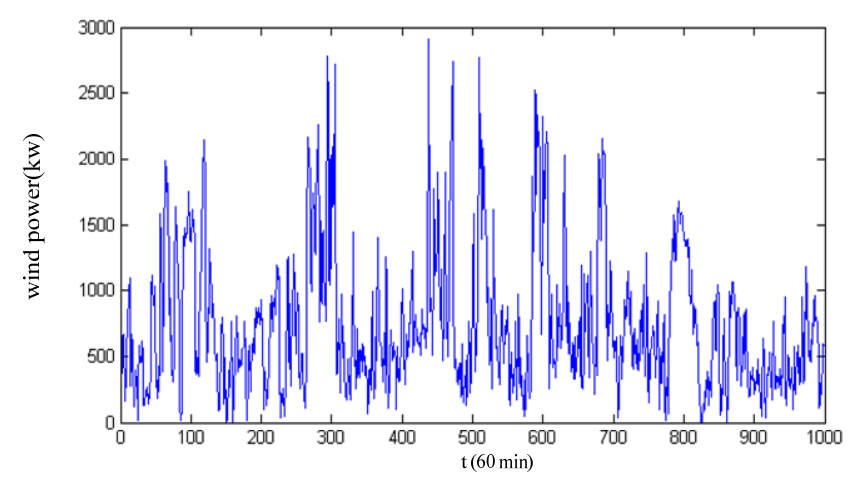

Fig. 2. The actual output power curve of a wind turbine.
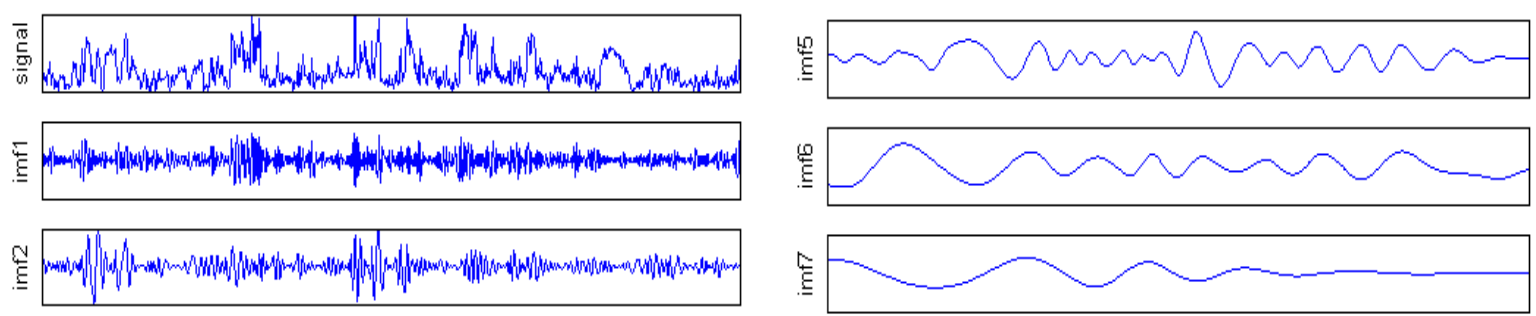

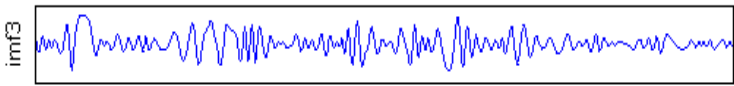

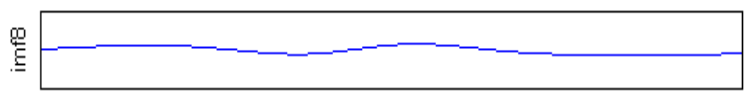

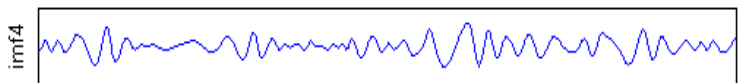

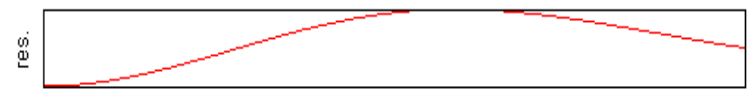

Fig. 3. The decomposed results of wind turbine power using EMD. 
Based on the above analysis, according to the RBFNN model that described in PART 3 to build a forecast model for all the IMFs and residue RES. All the models include three layers: input layer, hidden layer and output layer, and each input sample consists of three parameters: wind power, wind speed, and wind direction, using the method purposed by literature [20] to determine the number of hidden layer neurons. In our forecast model, choose 900 samples to train the model and use the rest 74 samples to test the model. And all the prediction results can be seen in Fig. 4.

Based on the above analysis, according to the RBFNN model that described in PART 3 to build a forecast model for all the IMFs and residue RES. All the models include three layers: input layer, hidden layer and output layer, and each input sample consists of three parameters: wind power, wind speed, and wind direction, using the method purposed by literature [20] to determine the number of hidden layer neurons. In our forecast model, choose 900 samples to train the model and use the rest 74 samples to test the model. And all the prediction results can be seen in Fig. 4 .
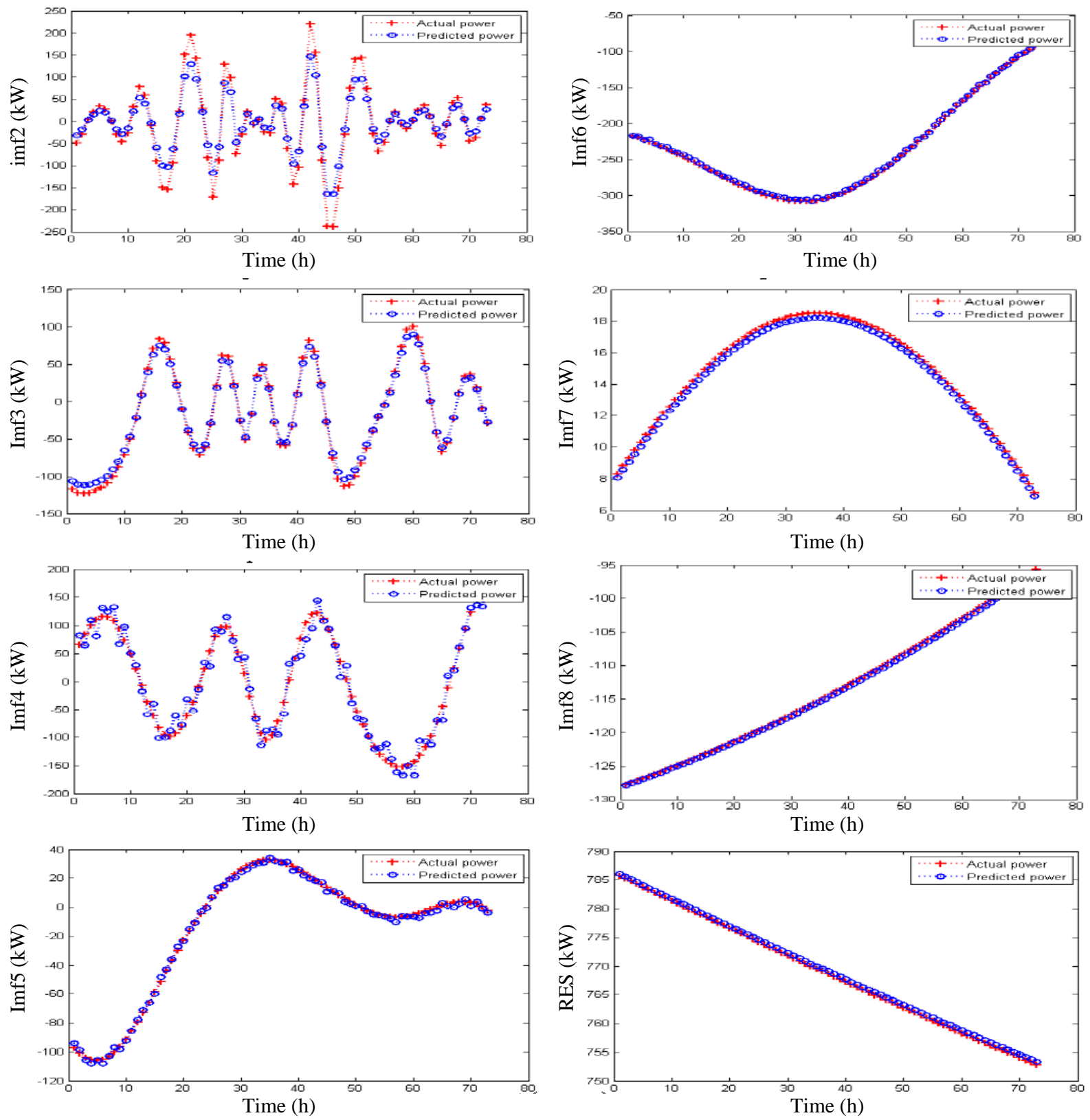

Fig. 4. Predicted power curve. 
Aggregate all the prediction components to obtain the final prediction value of wind power. In order to check the performance of the forecast model purposed in this paper, a model based on RBFNN has been constructed to test the same experimental data, and the prediction comparison can be seen in Fig. 5 and Table 1.

Table 1. The performance of two prediction methods.

\begin{tabular}{|l|l|l|l|l|}
\hline \multicolumn{1}{|c|}{ Prediction method } & MAE & MSE & MAPE(\%) & MSPE (\%) \\
\hline Prediction model based on EMD and RBFNN & 22.075 & 2.485 & 17.582 & 8.742 \\
\hline Prediction model based on RBFNN & 34.905 & 4.293 & 29.543 & 18.265 \\
\hline
\end{tabular}

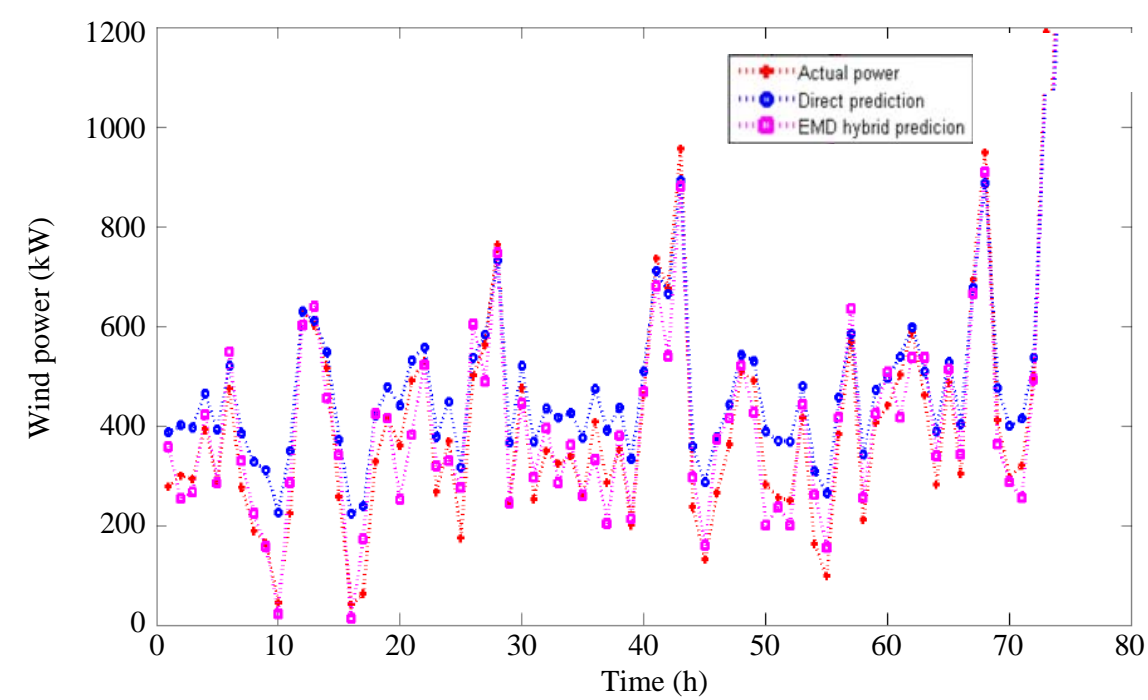

Fig. 5. The actual and predicted power by EMD and RBFNN prediction and direct prediction.

\section{Conclusion}

In this paper, a short-term wind power forecast method based on empirical mode decomposition (EMD) and radial basis function neural networks(RBFNN) is purposed. Decomposing wind power by EMD into components with different characteristics, reducing non-stationary and non-linear influence of wind power, which make these components are easier to analyze and predict compared to the original wind power analyses method. Then building RBFNN forecast models for each component and aggregating all the predictive values of the components to obtain the ultimate predictive result. After simulation of a wind power turbine, the results show the proposed prediction model has better prediction accuracy than the direct prediction method based on RBFNN. The simulation results also show the prediction model in this paper is an effective short-term wind power prediction method.

\section{Acknowledgements}

This work was supported by Natural Science Foundation of Zhejiang Province (Y1111254) and Zhejiang non-profit applied research projects (2010C31021).

\section{References}

[1] European Wind Energy Association (EA). (October 2002). Wind force $12 . \quad$ [Online]. Available: http://www.ewea.org/doc/WindForce12.pdf

[2] Louka P, Galanis G, Siebert N, et al. Improvements in wind speed forecasts for wind power prediction purposes using Kalman filtering. J. Wind Eng. Ind. Aerodyn, 2008; 96(12):2348-2362.

[3] Ramirez-Rosado I, Fernandez-Jimenez L, Monteiro C, Sousa J, Bessa R. Comparison of two new short-term wind-power forecasting systems. Renew Energy, 2009; 34(7):1848-54.

[4] El-Fouly THM, El-Saadany EF, Salama MMA. One day ahead prediction of wind speed using annual trends. IEEE Trans. on 
Energy Conversion, 2008; 23(1):191-201.

[5] Louka P, Galanis G, Katsafados GK, et al. Improvements in wind speed forecasts for wind power prediction purposes using kalman filtering. Journal of Window Engineering, 2005; 96(2):2348-2362.

[6] Dutta S, Overbye TJ. Prediction of short term power output of wind farms based on least squares method. In: Proc. of Power and Energy Society General Meeting, 2010 IEEE, 2010:1-6.

[7] Philippopoulos K, Deligiorgi D. Stochastic modeling of hourly average wind speed sequences in national observatory of athens. In: Proc. of the 9th Conference on Environmental Science and Technology, 2005:729-734.

[8] Juban J, Siebert N, Kariniotakis GN. Probabilistic short term wind power forecasting for the optimal management of wind generation. In: Proc. of Power Tech, IEEE Lausanne, 2007:683-688.

[9] Damousis IG, Alexiadis MC, Theocharis JB, Dokopoulos PS. A fuzzy model for wind speed prediction and power generation in wind parks using spatial correlation. IEEE Trans. Energy Convers., 2004; 19(2):352.

[10] Yiannis A. Katsigiannis, Antonis GT, et al. Improved wind power forecasting using a combined neuro-fuzzy and artificial neural network model. [Online]. Available: http://www.springerlink.com/content/3q077r6464518k36

[11] Mabel CM, Fernandez E. Analysis of wind power generation and prediction using ANN: a case study. Renewable Energy, 2008; 33(5):986-992.

[12] Negnevitsky M, Potter C. Innovative short-term wind generation prediction techniques. In: Proc. of the IEEE/PES General Meeting, 2006:18-22.

[13] Kitajima T, Yasuno T. Output prediction of wind power generation system using complex-valued neural network.. In: Proc. of SICE Annual Conference, 2010:3610-3613.

[14] Kariniotakis GN, Stavrakakis GS. Nogaret E. Wind power forecasting using advanced neural network models. IEEE Trans.On Energy Conversion, 1996; 11(4):762-767.

[15] Huang NE, Shen Z, Long SR, et al. The empirical mode decomposition and the Hilbert spectrum for nonlinear and nonstationary time series analysis. In: Proc.of the Royal Society of Longdon, Series A ,1998:903-995.

[16] Walker DM, Mees AI. Noise reduction of chaotic systems by Kalman filtering and by shadowing. Int. J. Bifur. Chaos, 1997; 7: pp.769-780.

[17] Amjad N, Keynia F. Day-ahead price forecasting of electricity markets by mutual information technique and cascaded neuroevolutionaryalgorithm. IEEE Trans. Power Syst., 2009; 24(1):306-318.

[18] Moody J, Darken CJ. Fast learning in networks of locally tuned processing units. Neural Computation, 1989; 1(2):281-294.

[19] Miller SJ. The Method of Least Squares, Brown University, Providence, 2006.

[20] Yun Z, Quan Z, Caixin S, Shaolan L, Yuming L, Yang S. RBF neural network and ANFIS-based short-term load forecasting approach in real-time price environment. IEEE Trans. on Power Systems, 2008; 23(3):853-858. 